\title{
La Gamificación como Predictor de la Integración en la Enseñanza
}

\section{Gamification as Predictor of the Integration in Teaching}

Blanca Antonia Rodríguez-Martínez ${ }^{I}$

\section{2}

\section{EDICIÓN: \#-CIVTAC}

Recibido: 25/junio/2021

Aceptado: $18 /$ septiembre/2021

Publicado: 24/septiembre/2021

我País

${ }^{1}$ Nicaragua

\section{IIIII Institución}

${ }^{1}$ Universidad Politécnica de Nicaragua

\section{Correo Eletrónico}

1ing.docente3@upoli.edu.ni

\section{(iD ORCID}

${ }^{1}$ https://orcid.org/0000-0003-4760-4621

\section{Citar así: G APA / IEEE}

Rodríguez-Martínez, B. (2021). La Gamificación como Predictores de la Integración en la Enseñanza. Revista Tecnológica-Educativa Docentes 2.0, 11(2), $\quad 57-65$ https://doi.org/10.37843/rted.v11i2.253

B. Rodríguez-Martínez, "La Gamificación como Predictores de la Integración en la Enseñanza", RTED, vol. 11, n. ${ }^{\circ}$ 2, pp. 5765 , sep. 2021.

\section{Resumen}

Actualmente, la educación superior demanda a los docentes innovar recursos didácticos integrando Tecnologías de la Información y la Comunicación (TIC) en la enseñanza para despertar el interés del estudiantado. El presente artículo el objetivo general fue Desarrollar la gamificación como estrategia didáctica en los estudiantes de primer semestre de la carrera Licenciatura en Derecho de Universidad Politécnica de Nicaragua, para el fortalecimiento del aprendizaje de la asignatura aplicaciones informáticas. En la metodología se empleó el paradigma interpretativo con un enfoque cualitativo, un tipo descriptivo aplicando el método inductivo-deductivo en el diseño Investigación-Acción con una muestra conformada por 60 estudiantes. Los resultados evidenciaron que el diseño de la propuesta de gamificación permitió la innovación de metodologías emergentes integrando TIC por medio de juegos educativos; la implementación de la gamificación favoreció a los estudiantes de la asignatura de aplicaciones informáticas una experiencia de aprendizaje placentera, y significativa, incrementando su interés-motivación por aprender de manera autónoma. Finalmente, se concluyó que la gamificación es de gran importancia en la educación superior porque permite el desarrollo de habilidades cognitivas y fortalece el proceso de enseñanza-aprendizaje, promoviendo ambientes de aprendizaje significativos que ayudan a enriquecer las competencias tecnológicas inherentes.

Palabras clave: Gamificación, predictores de la integración, TIC, enseñanza.

\section{Abstract}

Currently, higher education demands that teachers innovate didactic resources by integrating Information and Communication Technologies (ICT) in teaching to awaken students' interest. The general objective was to develop gamification as a didactic strategy in first semester students of the Bachelor of Law degree at the Polytechnic University of Nicaragua, to strengthen the learning of the subject computer applications. In the methodology, the interpretive paradigm was used with a qualitative approach, a descriptive type applying the inductive-deductive method in the Research-Action design with a sample of 60 students. The results showed that the creation of the gamification proposal included the innovation of emerging methodologies integrating ICT through educational games; The implementation of gamification gave the students of the computer applications subject a pleasant and meaningful learning experience, increasing their interest-motivation to learn autonomously. Finally, it concluded that gamification is of great importance in higher education because it allows the development of cognitive skills and strengthens the teaching-learning process, promoting meaningful learning environments that help enrich the inherent technological competencies.

Keywords: Gamification, predictors of integration, ICT, teaching. 


\section{Introducción}

Actualmente, la educación superior demanda a los docentes innovar recursos didácticos integrando Tecnologías de la Información y la Comunicación (TIC) en la enseñanza para despertar el interés del estudiantado, cambiar la percepción de asignaturas como aburridas porque los nuevos estudiantes que ingresan a la universidad son jóvenes nacidos, criados con tecnología, con una habilidad innata del lenguaje, del entorno digital donde las herramientas tecnológicas ocupan un lugar central en su forma de relacionarse, comportarse, estudiar, entre otros, por lo tanto, el desafío radica en el empleo de estrategias de enseñanza creativas para transmitir conocimiento e incentivar aprendizaje.

Aunado a esto, los docentes universitarios son los más afectados en la utilización de los nuevos avances tecnológicos. Al respecto, Fonoll-Salvador et al. sostiene: "para el profesor las tecnologías digitales suponen un cambio en su forma de trabajar, cambios metodológicos en su planteamiento de actuación en el aula. Esto significa, el profesor debe adaptar los contenidos educativos a las necesidades del alumno" (2011, p.36). Debido a esto, surge la necesidad de planificar estrategias de enseñanza innovadoras suficientemente llamativas para lograr en los estudiantes no solamente almacenen conceptos, sino que maduren la capacidad de entenderlos y servirse de ellos para solucionar problemas (Melo-Herrera \& Hernández-Barbosa, 2014).

La dificultad radica en ¿cómo efectuar la implementación de estrategias de enseñanza innovadoras para propiciar el aprendizaje significativo en los nativos digitales? Giró-Miranda (1998) señala a los juegos educativos como herramientas didácticas aprovechables para conseguir aprendizaje porque el docente transforma los contenidos de manera creativa para acomodarlos a los objetivos pedagógicosformativos. En este sentido, Oliva (2017) plantea la gamificación como una estrategia metodológica útil en la mejora docente para incidir en el estudiantado de manera positiva en la consecución de objetivos de aprendizajes.

Ante lo expuesto, el objetivo general de la investigación radica en desarrollar gamificación como estrategia didáctica en los estudiantes de primer semestre de la carrera Licenciatura en Derecho de Universidad Politécnica de Nicaragua para el fortalecimiento del aprendizaje de la asignatura aplicaciones informáticas. Asimismo, se platean los objetivos específicos: Indagar referentes teóricos de gamificación; Diseñar la propuesta de gamificación mediante juegos educativos; Implementar la gamificación en la plataforma Educaplay en la asignatura aplicaciones informáticas.

\section{Metodología}

La presente investigación se desarrolló bajo el paradigma interpretativo con un enfoque cualitativo. Esto permitió comprender el mundo de experiencia vivida desde el punto de vista de personas (Taylor \& Bogdan, 1987), además exploró el fenómeno desde perspectiva de los participantes en su ambiente natural en relación con el contexto, con el fin de profundizar en sus puntos de vistas, interpretaciones, significados (Hernández-Sampieri \& Mendoza-Torres, 2018). De este modo, el enfoque cualitativo permitió indagar las necesidades del estudiantado de primer semestre de la Licenciatura en Derecho en relación con proceso de enseñanza-aprendizaje e interpretarlo para evidenciar los resultados sobre los saberes alcanzados en función de una estrategia didáctica innovadora mediante gamificación, empleando una herramienta TIC online de código abierto como es Educaplay.

El estudio de investigación se enmarcó en los fundamentos correspondiente al tipo descriptivo porque caracteriza al grupo social por medio de observación aplicando el método inductivo-deductivo a través del análisis de unidades de significado. Además, se basó en el diseño Investigación-Acción donde la investigadora interpreta la vida en el aula de clase teniendo un posicionamiento no 
neutral ni distante interviniendo junto con los estudiantes. Restrepo Gómez (2004) declara la investigación-acción en el ámbito educativo es un instrumento de ejercicio reflexivo que permite al maestro comportarse como aprendiz de largo alcance comprendiendo la estructura del quehacer académico y transformando sistemáticamente la práctica pedagógica.

La población estuvo constituida por 60 estudiantes de primer semestre de la carrera Licenciatura en Derecho de Universidad Politécnica de Nicaragua. De acuerdo con la población mencionada, se tomó como muestreo intencional a 10 estudiantes para una encuesta con la cual se logró obtener información sobre las debilidades de la primera unidad titulada "introducción a informática". Posteriormente, se diseñó una serie de juegos educativos considerando los contenidos a tratar en cada sesión de clase con el objetivo de fortalecer los vacíos arrojados por la encuesta. Finalmente, en la implementación de estos se usó la plataforma de gamificación Educaplay que es un servicio web educativo para aprender divirtiéndose, donde la docente creó y compartió los juegos haciendo la incrustación del iframe (código html) en el curso del entorno virtual de aprendizaje de la institución.

Por otra parte, los estudiantes ingresaron haciendo uso de dispositivo electrónico (computadora, celular) al curso para realizar actividades didácticas con interfaces intuitivas que permita su fácil manejo. Esto favorece su uso, el alumno percibe como juegos, no como un sistema de evaluación, siendo evidente la motivación intrínseca cada vez que participa en ellos porque aprende jugando (Fernández et al, 2016).

\section{Resultados}

La gamificación es un conjunto instruccional de juego para la construcción de conocimiento aplicados en un contexto. Según Kapp (2012) la gamificación sirve a los docentes como una técnica significativa que ayuda potencialmente al proceso enseñanza- aprendizaje. Al respecto Teixes (2015) considera que la gamificación "es la aplicación de recursos propios de juegos (diseño, dinámica, elementos, etc.) en contextos no lúdicos, con el fin de modificar los comportamientos de los individuos, actuando sobre su motivación, para consecución de objetivos concretos" (p.18). Asimismo, Borras (2015) señala que el juego es pieza indispensable para gamificar las actividades a efectuarse en el aula. Con estas apreciaciones, se evidencia la utilidad de la gamificación para propiciar el aprendizaje significativo del estudiantado.

En el ámbito educativo, cada vez se incorpora gamificación como herramienta en el proceso enseñanza-aprendizaje para promover la participación del estudiantado en actividades académicas por medio del diseño de juegos. Estos se caracterizan según Faiella $\&$ Ricciardi en "ofrecer la posibilidad de reformular el fracaso como una parte necesaria del aprendizaje, puesto que el error se convierte en una oportunidad de probar, para practicar y mejorar" (2015, p.18). Además, los juegos mejoran la capacidad de enseñanza en el aula de clase, porque es un elemento importante en la resolución de problemas (Morris et al., 2013), siendo indiscutible el poder de la motivación (Gianneto et al.,2013; Simões et al., 2013). Por tanto, los docentes buscan herramientas digitales que faciliten la participación del estudiante en su experiencia de aprendizaje.

La gamificación por medio de herramientas TIC complementa y enriquece el sistema educativo. En tal sentido, Grass-Martí et al. (2005) afirma: "la adquisición de conocimientos y habilidades relacionadas con las TIC, y su aplicación en las tareas docentes representan un reto que exige replantear e integrar los contenidos y la puesta en práctica de metodologías nuevas en el proceso de enseñanza-aprendizaje" (p.2). Es así como, el empleo de las TIC a través de juegos vislumbra las potencialidades de la enseñanza por lo tanto son usadas como "estrategias pedagógicas, brindan la posibilidad de crear oportunidades para guiar e incrementar el aprendizaje y colaboran al docente a llevar a 
cabo procesos innovadores" (Pedraza, 2009, p.321).

Hoy en día existen un sin número de plataformas educativas TIC online de acceso gratuito como Educaplay. Esta es una herramienta gamificada que permite diseñar e implementar juegos (mapas, adivinanzas, dictados, crucigrama, sopa de letras, etc.) para el uso docente. Este asume la responsabilidad de estudiarlas, apropiarse de su manejo con el fin de seleccionar idóneamente en función de sus intereses alineado a las necesidades de aprendizaje del estudiantado.

Así pues, una planificación didáctica basada en la herramienta Educaplay ayuda a mantener el interés de los estudiantes en aprender jugando y evita que el proceso enseñanza-aprendizaje se convierta en algo monótono y cansado. Valderrama (2015) señala la importancia del juego como una actividad intrínsecamente motivadora en la que los estudiantes aprenden y desarrollan habilidades de inteligencia emocional y social. En esta misma línea, Scott \& Neustaedter (2013) recogen cuatros beneficios de los juegos en la gamificación: libertad para fallar, rápido feedback, progreso, historia por lo que la implementación en un ambiente educativo (Perrota et al., 2013) ayuda al profesor a trabajar en un contexto de persuasión e invitación en lugar de obligación haciendo la conexión de los contenidos entre sí con el fin de dar sentido a lo que se está trabajando en cada momento.

Finalmente, diseñar gamificación como estrategia didáctica permite recurrir a los elementos característicos del juego y así poder implementar la herramienta gamificada Educaplay de manera eficiente en el aula de clase. Por otra parte, es importante reconocer que no todos los estudiantes tienen los mismos intereses siendo indispensable identificar las motivaciones de los participantes para desarrollar un ambiente atractivo de aprendizaje que propicien en mayor medida el involucramiento y el consecuente desenvolvimiento de todos los participantes en las actividades educativas (Aranda Romo \& Caldera Montes, 2018).
La gamificación integrada por TIC (Educaplay) en el ámbito de enseñanza se convirtió en una experiencia significativa fundamentada en factores como la motivación e identificación social a través de los juegos educativos dinamizadores, atractivos donde los estudiantes alcanzaron un mayor compromiso en su aprendizaje porque se divirtieron mientras lo hacían. Lo anterior confirmó que el juego educativo es un proceso positivo que permite combinar modernas metodologías hábiles viables para educación posibilitando en los alumnos acciones experimentales, donde pueden recrear, reintentar, observar e innovar para aprender (Álvarez \& Polanco, 2018).

Los juegos educativos fueron diseñados para tener una duración que variaba entre los 5 minutos y los 20 minutos según la sesión de clase en que se aplicaba. A continuación, se describe detalladamente estos en las cuatro sesiones de clases de la primera unidad:

Primera sesión. Empleó dos juegos: sopa de letras elementos informáticos como se muestra en la Figura 1 para los estudiantes encuentren los conceptos planteados. Mapa interactivo como se muestra en la Figura 2 para los estudiantes reconozca el nombre con la figura asociada.

\section{Figura 1}

Sopa de letras elementos informáticos

\begin{tabular}{|lllllllllllll|l|l|l|} 
I & N & F & O & R & M & A & T & I & C & A & Q & Q & J & 1. COMPUTADORA \\
P & S & I & X & H & S & N & D & B & C & N & B & F & L & 2. INFORMACION \\
I & U & V & H & O & S & O & F & T & W & A & R & E & V & 3. INFORMATICA \\
H & Y & C & E & N & D & I & B & J & X & A & L & S & Y & 4. APLICACION \\
E & V & O & H & U & U & C & I & Q & J & A & K & X & Y & 5. HARDWARE \\
R & C & M & E & U & J & A & E & J & A & P & O & H & V & 6. SOFTWARE \\
A & J & P & D & M & C & M & F & Q & X & L & V & O & O & 7. DATO \\
W & Y & U & M & G & H & R & J & A & E & I & X & L & I & \\
D & Y & T & Q & L & P & O & G & O & A & C & Y & C & Q & \\
R & Q & A & S & D & M & F & F & M & I & A & P & K & F & \\
A & R & D & S & Y & J & N & K & H & D & C & A & T & D & \\
H & L & O & P & I & F & I & J & T & A & I & I & S & S & \\
J & S & R & D & S & E & V & B & N & T & O & N & S & W & \\
P & O & A & H & B & G & I & O & F & O & N & Q & Q & F & &
\end{tabular}

Nota. Opciones de respuesta de sopa de letras elementos informáticos, elaborado por RodríguezMartínez (2021). 


\section{Figura 2}

Mapa interactivo dispositivos E/S

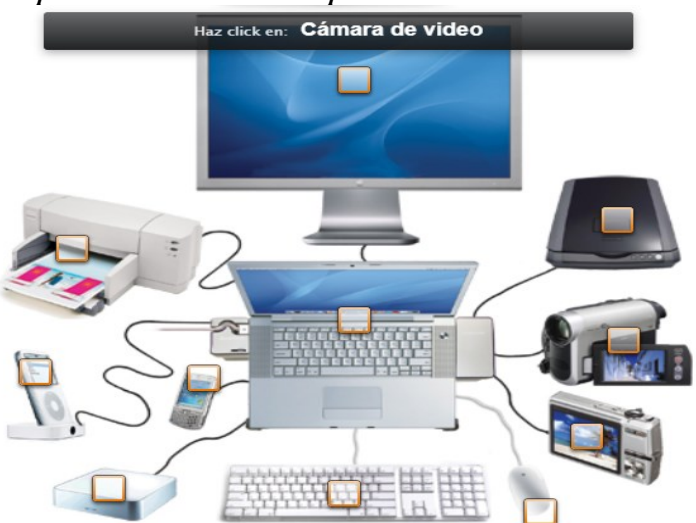

Nota. Dispositivos Entrada/Salida electrónicos, elaborado por Rodríguez-Martínez (2021).
Segunda sesión. Empleó dos juegos: relacionar columnas generación de computadoras como se muestra en la Figura 3 para los estudiantes identifiquen el aspecto de generación de computadora con figura asociada. Relacionar grupos tipos de software como se muestra en la Figura 4 para los estudiantes asocien el tipo de software (software de sistema, software de aplicación, software de programación) haciendo clic en el nombre incorporado.

\section{Figura 3}

Relacionar columnas generación de computadora

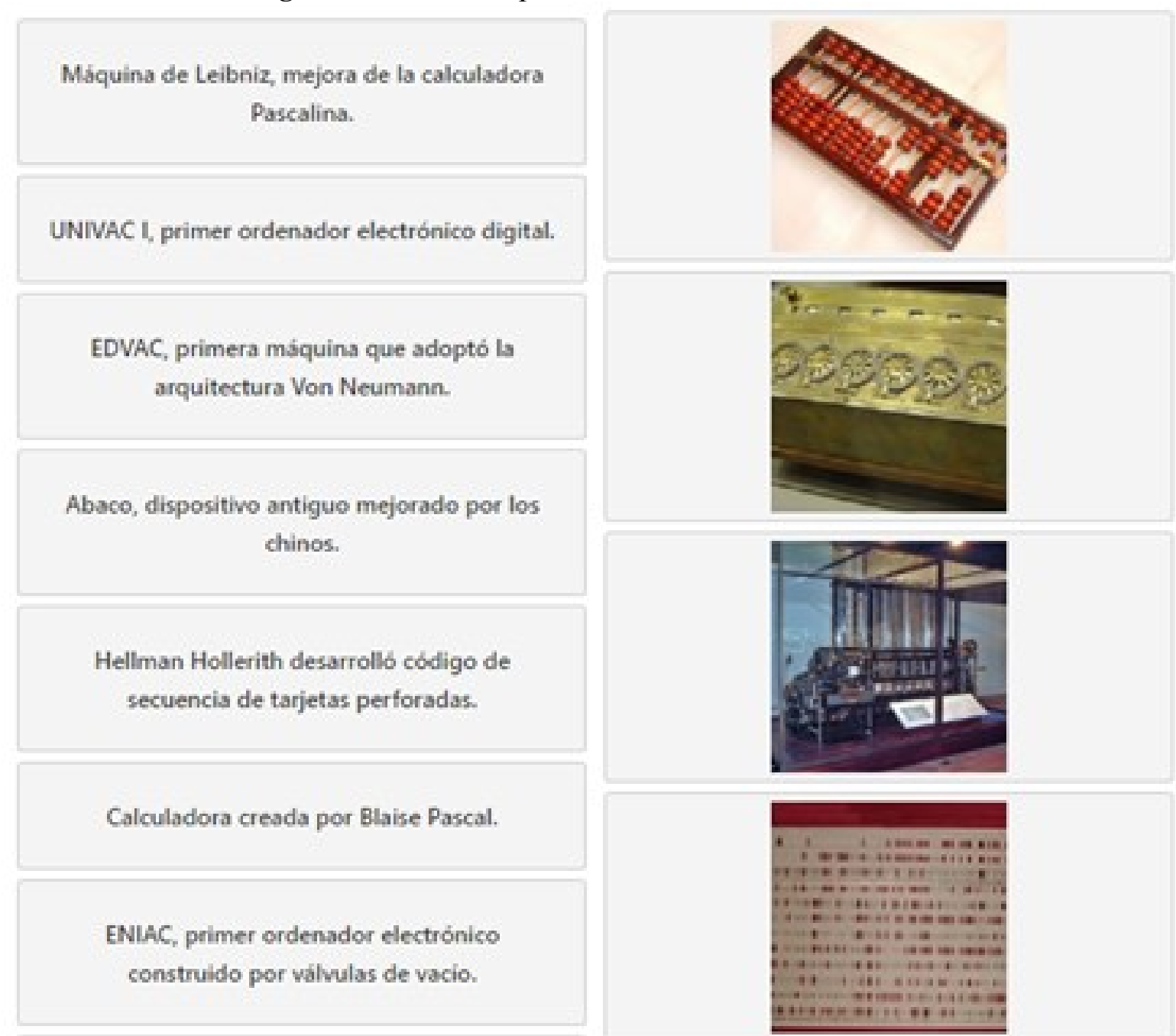

Nota. Relacionar columnas generación de computadoras, elaborado por Rodríguez-Martínez (2021). 


\section{Figura 4}

Relacionar el tipo de software

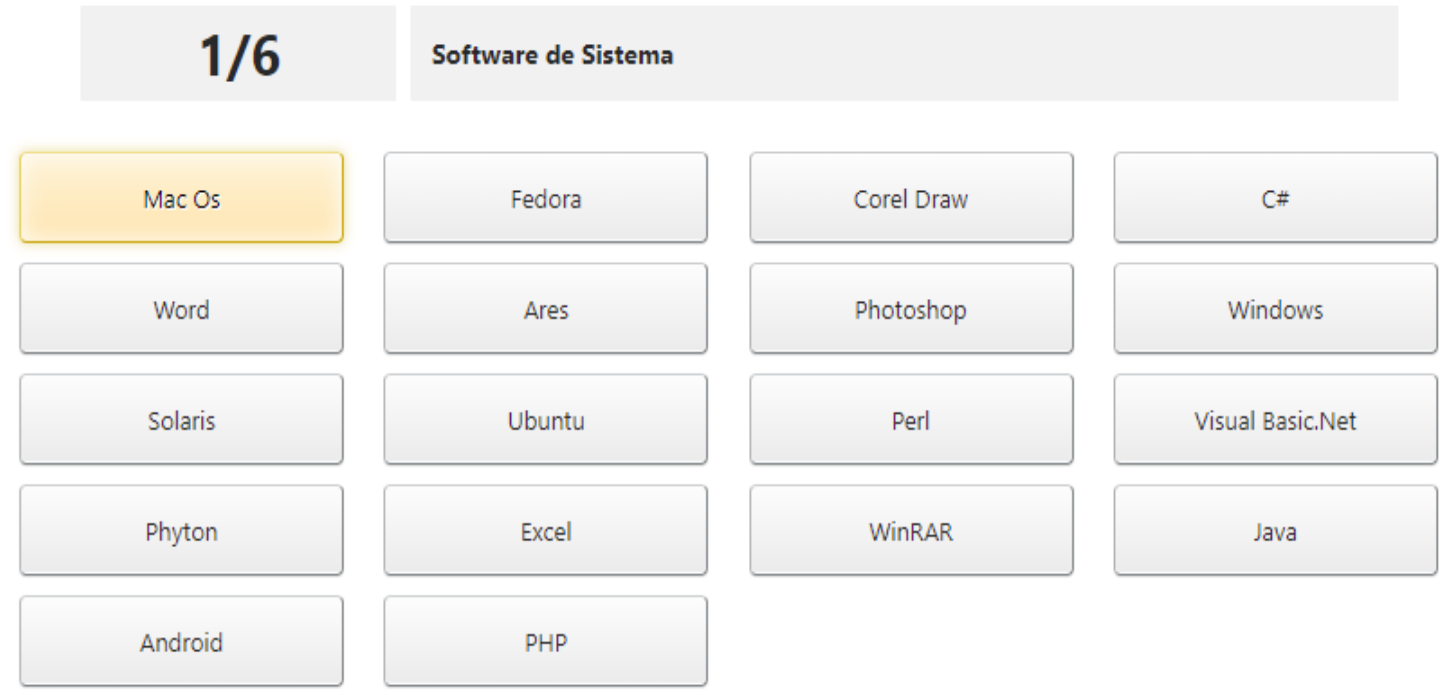

Nota. Relacionar el tipo de software, elaborado por Rodríguez-Martínez (2021).

Tercera sesión. Empleó dos juegos: mapa interactivo hardware interno como se muestra en la Figura 5 para los estudiantes asocien el nombre del dispositivo interno con figura asociada. Mapa interactivo hardware externo como se muestra en la Figura 6 para los estudiantes asocien el nombre del dispositivo externo con figura asociada.

\section{Figura 5}

\section{Mapa interactivo hardware interno}

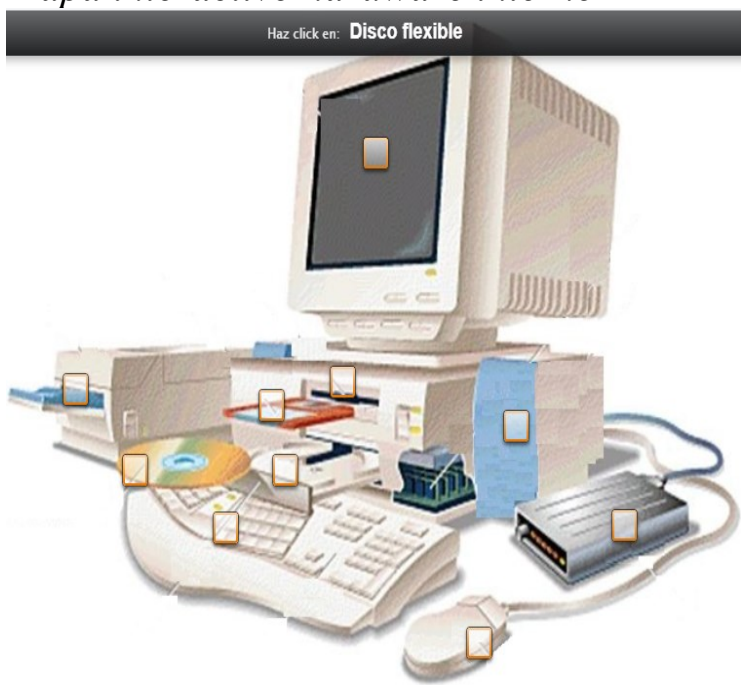

Nota. Hardware interno, elaborado por RodríguezMartínez (2021).

\section{Figura 6}

Mapa interactivo hardware externo

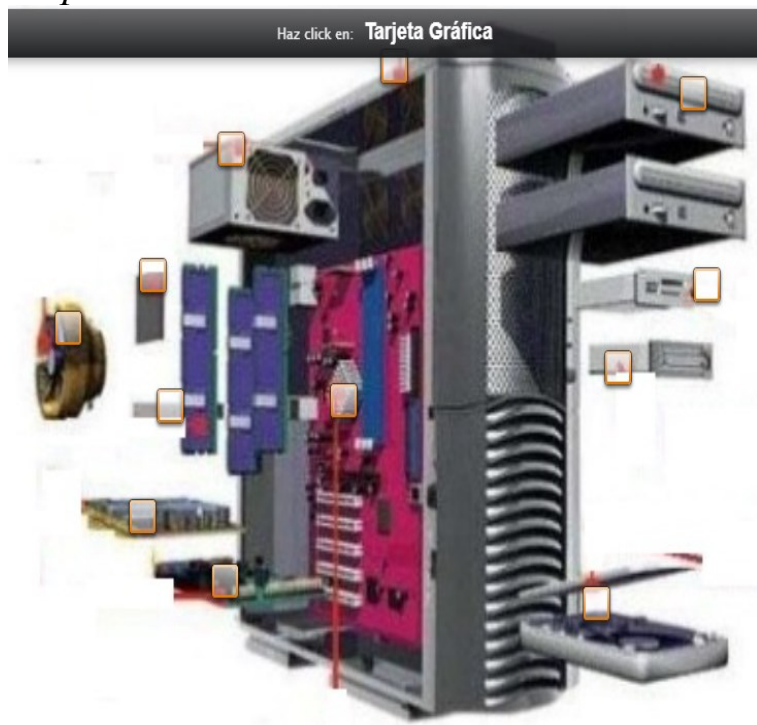

Nota. Hardware externo, elaborado por RodríguezMartínez (2021).

Cuarta sesión: Empleó el juego Test conversiones de almacenamiento como se muestra en la Figura 7a y Figura $7 \mathrm{~b}$ para los estudiantes seleccionen dentro de unidades de almacenamiento la conversión correcta. Solo se va a mostrar dos preguntas de 15 del juego educativo. 


\section{Figura 7a}

Test conversiones de almacenamiento

5. ¿Conversiones de Almacenamiento

Anterior

Nota. Conversiones de almacenamiento pregunta 5, elaborado por Rodríguez-Martínez (2021).

\section{Figura $7 \mathbf{b}$}

Test conversiones de almacenamiento

10. En una memoria USB de 2 GB se copia 320 fotos de 288 KB cada una, 100 videos MP4 de $10 \mathrm{MB}$ cada uno. ¿Cuánto es el espacio ocupado de la memoria USB en MB?

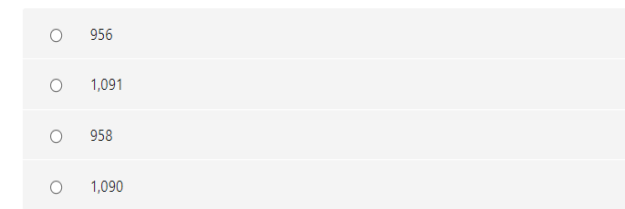

Anterior

Nota. Conversiones de almacenamiento pregunta 10 , elaborado por Rodríguez-Martínez (2021).

\section{Discusión}

Implementar gamificación en la plataforma Educaplay con los estudiantes de primer semestre de la Licenciatura en Derecho proporcionó la consecución de los objetivos de aprendizaje planteados por la docente. Kaufmann (2018) y Majuri et al. (2018) enfatizan que la gamificación ayuda a los estudiantes a superar desafíos académicos. Asimismo, la investigación realizada por Chung et al. (2019) determinaron el impacto efectivo de la gamificación en la mejora del rendimiento académico, expectativa de esfuerzo, influencia social y apropiación de conocimientos entonces los estudiantes con un sentimiento positivo sobre la utilidad de la gamificación tendrán más intención de usarlo (Tarhini et al., 2016).

En tal sentido, la propuesta de investigación se podría replicar en otras asignaturas y carreras de la Universidad Politécnica de Nicaragua al incorporar los juegos educativos en un ambiente atractivo para el estudiantado. Los resultados de las investigaciones presentadas por Subhash \& Cudney (2018) y Pertegal \& Lledó (2019) evidencian los beneficios de incluir en la planificación pedagógica gamificación porque posibilita reactivar la atención de los estudiantes, desarrollo de habilidades cognitivas, afianzamiento de los conocimientos, autoevaluación y retroalimentación in situ.

\section{Conclusiones}

La indagación de gamificación se llevó a cabo mediante el análisis documental de literatura existente. Esto permitió examinar estudios empíricos exitosos de gamificación donde los beneficios dependen del dominio de los elementos del juego y correcta aplicación de herramienta TIC. Además, diseñar la propuesta de gamificación mediante juegos educativos posibilitó a la docente incorporar distintos recursos tecnológicos que permitan mejorar el aprendizaje en los estudiantes.

Implementar la gamificación en la plataforma Educaplay en la asignatura aplicaciones informáticas fue sin duda una estrategia didáctica innovadora tanto para docente y estudiantes, dado el aprendizaje se realizó mediante juegos educativos permitieron afianzar los conocimientos. En efecto, esta herramienta TIC de software gratuito online Educaplay facultó incrustar iFrame en la página del curso para acceder directamente a los juegos evitando inconvenientes de subirlos directamente y evitar ralentizar el sitio.

La aplicación en sesiones de clases evidenció en los estudiantes actitudes motivadoras, mayor concentración en el momento de responder, atraer interés de los estudiantes en participar, acertar en cada una de respuestas del juego educativo para lograr puntajes, reconocimientos. Finalmente, la docente pudo constatar en tiempo real influencia, efectos positivos de la gamificación en el proceso de enseñanzaaprendizaje porque dinamizó sesiones de clases, reforzó apropiación de contenidos de los estudiantes. Así mismo, los estudiantes acogieron positivamente la gamificación por ser una experiencia placentera, entretenida de 
aprender jugando, donde ellos pueden practicar en cualquier lugar, horario reforzando el material e incentivando el aprendizaje autónomo.

Esta investigación puede servir como objeto de reflexión para los docentes con el propósito de incentivar el uso de la gamificación en el aula porque beneficia al estudiantado en su proceso de aprendizaje. Por otro lado, es necesario futuras investigaciones para medir y cuantificar la mejora en el rendimiento académico.

\section{Reconocimiento}

Quiero agradecer primeramente a Dios por su Misericordia, a mi madre por su dedicación, sus consejos, los valores inculcados durante mi vida me ha formado, ayudado a crecer como persona. Además, extiendo mi agradecimiento a autoridades, estudiantes de la carrera de Licenciatura en Derecho por permitir este estudio se lleve a cabo.

\section{Referencias}

Álvarez, A., \& Polanco, N. (2018). La gamificación como experiencia de aprendizaje en la educación. Revista Tecnológica Educativa Docentes 2.0, 6(3), 39. https://ojs.docentes20.com/index.php/revistadocentes $20 /$ article/view $/ 30$

Aranda-Romo, M. G., \& Caldera-Montes, J. F. (2018). Gamificar el aula como estrategia para fomentar habilidades socioemocionales. Revista educarnos, 8(31), 41-66. https://revistaeducarnos.com/wpcontent/uploads/2018/09/articulo-mariaguadalupe.pdf

Borras, O. (2015). Fundamentos de la gamificación. Universidad Politécnica de Madrid.

Chung, C.H., Shen, C. \& Qiu, Y.Z. (2019). Students' Acceptance of Gamification in Higher Education. International Journal of Game-Based Learning, $9(2)$,

19. https://doi.org/10.4018/IJGBL.2019040101

Faiella, F., \& Ricciardi, M. (2015). Gamification and learning: A review of issues and research. Journal of ELearningand Knowledge Society, 11(3), 1321.

https://www.researchgate.net/publication/2837575 60_Gamification_and_learning_A_review_of_iss ues_and_research

Fernández, A., Olmos, J. \& Alegre, J. (2016). Valor pedagógico del repositorio común de conocimientos para cursos de dirección de empresas. Revista Educativa. https://www.redalyc.org/pdf/3495/349546195005. pdf
Fonoll-Salvador, J., Garcia-Fernández, J., Garcia-Villalobos, J., Guerra-Álvarez, A., Gutiérrez-Restrepo, E., Jaúdenes-Casaubón, C., Martínez-Normand, L., \& Romero-Zúnica, R. (2011). Accesibilidad, TIC y educación. Ministerio de Educación, Cultura y Deporte de España. https://www.digitaliapublishing.com/a/14716/acce sibilidad--tic-y-educacion

Gras-Martí, A., Cano-Villalba, M., Soler-Selva, V. \& Santos Benito, J. (2005). Aprovechamiento de recursos TIC para mejorar el aprendizaje de los lenguajes de las Ciencias. Investigaciones didácticas en el aula. Revista Digital de Investigación en Docencia Universitaria

1(1),1-14. https://doi.org/10.19083/ridu.1.34

Hernández-Sampieri, R. \& Mendoza-Torres, C. P. (2018). Metodología de la investigación. Las rutas cuantitativa, cualitativa y mixta. McGraw Hill.

Kapp, K. (2012). The gamification of learning and instruction: game-based methods and strategies for training and education. John Wiley \& Sons, Inc.

Kaufmann, D. A. (2018). Reflection: Benefits of Gamification in Online Higher Education. IJournal of Instructional Research, 7, 125-132. https://files.eric.ed.gov/fulltext/EJ1188367.pdf

Majuri, J., Koivisto, J., \& Hamari, J. (2018). Gamification of education and learning: A review of empirical literature. In J. Koivisto, \& J. Hamari (Eds.), Proceedings of the 2nd International GamiFIN Conference, GamiFIN 2018 (Vol. 2186, pp. 11-19). (CEUR Workshop Proceedings; Vol. 2186, No. 2186). CEUR-WS. http://ceurws.org/Vol-2186/paper2.pdf

Melo-Herrera, M.P. \& Hernández Barbosa, R. (2014). El juego y sus posibilidades en la enseñanza de las ciencias naturales. Innovación Educativa, 14(66), 41-63.

https://www.redalyc.org/pdf/1794/179433435004 pdf

Morris, B. J., Croker, S., Zimmerman, C., Gill, D., \& Romig, C. (2013). Gaming science: The "Gamification" of scientific thinking. Frontiers in Psychology, 4. https://doi.org/10.3389/fpsyg.2013.00607

Oliva, H. A. (2017). La gamificación como estrategia metodológica en el contexto educativo universitario. Realidad y Reflexión (44), 29-47. https://doi.org/10.5377/ryr.v44i0.3563

Pedraza, Y. (2009). Experiencia de la enseñanza de la química con el apoyo de las TIC. Revista Educación Química, 20(3),320-329. http://www.scielo.org.mx/pdf/eq/v20n3/v20n3a4.p df

Restrepo-Gómez, B. (2004). La investigación-acción educativa y la construcción de saber pedagógico. Educación y Educadores, (7), 45-55. Universidad de La Sabana Cundinamarca. https://www.redalyc.org/pdf/834/83400706.pdf 
Simões, J., Redondo, R. D., \& Vilas, A. F. (2013). A social gamification framework for a K-6 learning platform. Computers in Human Behavior, 29(2), 345-353.

https://doi.org/10.1016/j.chb.2012.06.007

Subhash, S., \& Cudney, E. (2018). Gamified learning in higher education: A systematic review of the literature. Comput. Hum. Behav., 87, 192-206. https://www.sciencedirect.com/science/article/abs/ pii/S0747563218302541?via\%3Dihub

Tarhini, A., Hone, K., Liu, X., \& Tarhini, T. (2016). Examining the moderating effect of individuallevel cultural values on users' acceptance of Elearning in developing countries: a structural equation modeling of an extended technology acceptance model. Interactive Learning Environments, 25(3), 306-328. https://doi.org/10.1080/10494820.2015.1122635

Taylor, S.J. \& Bogdan, R. (1987). Introducción a los métodos cualitativos de investigación. Paidos

Teixes, F. (2015). Gamificación: fundamentos y aplicaciones. Editorial UOC.

Valderrama, B. (2015). Los secretos de la gamificación: 10 motivos para jugar. Capital Humano, 295, 73-78. https://www.researchgate.net/publication/2828698 61_Los_secretos_de_la_gamificacion 